\title{
Burst properties of the highly active FRB20201124A using uGMRT
}

\author{
V. R. Marthi ${ }^{\circledR},{ }^{1 \star}$ S. Bethapudi, ${ }^{2}$ R. A. Main, ${ }^{2}$ H.-H. Lin ${ }^{\circledR},{ }^{3,4}$ L. G. Spitler, ${ }^{2}$ R. S. Wharton, ${ }^{5}$ D. Z. Li, ${ }^{6}$ \\ T. Gautam, ${ }^{2}$ U.-L. Pen ${ }^{3,4,7,8,9}$ and G. H. Hilmarsson ${ }^{\oplus 2}$ \\ ${ }^{1}$ National Centre for Radio Astrophysics, Tata Institute of Fundamental Research, Post Bag 3, Ganeshkhind, Pune 411 007, India \\ ${ }^{2}$ Max-Planck-Institut für Radioastronomie, Auf dem Hügel 69, D-53121 Bonn, Germany \\ ${ }^{3}$ Institute of Astronomy and Astrophysics, Academia Sinica, Astronomy-Mathematics Building, No. 1, Section 4, Roosevelt Road, Taipei 10617, Taiwan \\ ${ }^{4}$ Canadian Institute for Theoretical Astrophysics, University of Toronto, 60 St. George Street, Toronto, ON M5S 3H8, Canada \\ ${ }^{5}$ NASA Postdoctoral Program Fellow, Jet Propulsion Laboratory, California Institute of Technology, Pasadena, CA 91109, USA \\ ${ }^{6}$ Cahill Center for Astronomy and Astrophysics, California Institute of Technology, MC 249-17, Pasadena, CA 91125, USA \\ ${ }^{7}$ Dunlap Institute for Astronomy and Astrophysics, University of Toronto, 50 St. George Street, Toronto, ON M5S 3H4, Canada \\ ${ }^{8}$ Program in Cosmology and Gravitation, Canadian Institute for Advanced Research, Toronto, ON M5G 1Z8, Canada \\ ${ }^{9}$ Perimeter Institute for Theoretical Physics, 31 Caroline Street North, Waterloo, ON N2L 2Y5, Canada
}

Accepted 2021 October 14. Received 2021 September 29; in original form 2021 August 1

\begin{abstract}
We report the observations of the highly active FRB20201124A with the upgraded Giant Metrewave Radio Telescope (uGMRT) at $550-750 \mathrm{MHz}$. These observations in the incoherent array mode simultaneously provided an arcsecond localization of bursts from FRB20201124A, the discovery of persistent radio emission associated with the host galaxy, and the detection of 48 bursts. Using the brightest burst in the sample $(F=108 \mathrm{Jy} \mathrm{ms})$, we find a structure-maximizing dispersion measure of $410.8 \pm 0.5 \mathrm{pc} \mathrm{cm}^{-3}$. We find that our observations are complete down to a fluence level of $10 \mathrm{Jy} \mathrm{ms}$, above which the cumulative burst rate scales as a power law $R(>F)=10 \mathrm{~h}^{-1}(F / 10 \mathrm{Jy} \mathrm{ms})^{\gamma}$, with $\gamma=-1.2 \pm 0.2$. We find that the bursts are, on average, wider than those reported for other repeating fast radio bursts (FRBs). We find that the waiting time between bursts is well approximated by an exponential distribution with a mean of $\sim 2.9$ min during our observations. We searched for periodicities using both a standard Fourier domain method and the fast folding algorithm, but found no significant candidates. We measure bulk spectro-temporal drift rates between -0.75 and $-20 \mathrm{MHz} \mathrm{ms}^{-1}$. Finally, we use the brightest burst to set an upper limit to the scattering time of $11.1 \mathrm{~ms}$ at $550 \mathrm{MHz}$. The localization of FRB20201124A adds strength to the proof-of-concept method described in our earlier work and serves as a potential model for future localizations and follow-up of repeating FRBs with the uGMRT.
\end{abstract}

Key words: scattering-methods: observational-techniques: interferometric.

\section{INTRODUCTION}

Fast radio bursts (FRBs) are short-duration (100 $\mu \mathrm{s}-100 \mathrm{~ms})$ radio flashes that are extremely bright and appear highly dispersed, and hence thought to be arising from extragalactic distances. Although a promising Galactic analogue has been found in SGR $1935+2154$ (Bochenek et al. 2020; CHIME/FRB Collaboration et al. 2020b), the origin of these enigmatic bursts remains a mystery. A wide range of FRB models have been proposed (see e.g. Platts et al. 2019), but none have been conclusively proven. It is not even known yet if there is a single class of FRB progenitor or multiple different ones that produce a similar observational phenomenon.

The discovery of repeating FRBs (Scholz et al. 2016; Spitler et al. 2016) marked a paradigm shift in FRB research. Repeaters provide an enormous opportunity to study individual FRB sources in great detail. Collecting a large number of bursts from a given source can reveal interesting burst structure (e.g. Hessels et al. 2019). Furthermore, since the dispersion measure (DM) of the bursts is known for repeaters, data from observations can be coherently

^E-mail: vrmarthi@ncra.tifr.res.in dedispersed. This allows us to examine the bursts at extremely high time resolution to explore the emission mechanism and constrain progenitor models (Majid et al. 2021; Nimmo et al. 2021). Repeating FRBs also allow for periodicity searches on a wide range of timescales. The discovery of a 16.35-d cycle in the burst activity of FRB20180916B (CHIME/FRB Collaboration et al. 2020a) not only provides an important insight into the progenitor source of FRBs, it also allows for highly efficient targeted observations (e.g. Marthi et al. 2020) to be conducted at the precise times at which the source is most likely to be active. A much longer activity cycle has also been proposed for FRB20121102A (Rajwade et al. 2020; Cruces et al. 2021), although it remains to be seen if any other repeaters show this cyclic activity. Repeating FRBs are also useful targets for searching for short time-scale periodicities of the order of $\sim 1 \mathrm{~ms}$ to $1 \mathrm{~s}$, which would indicate a neutron star origin. No pulsar-like periodicity has been detected in any FRBs (Zhang et al. 2018; Li et al. 2021), but CHIME/FRB recently discovered several FRBs with sub-second periodic separations of burst components (The CHIME/FRB Collaboration et al. 2021). This result provides further evidence in favour of a neutron star origin for FRBs and strongly motivates deep searches for pulsar-like periodic emission in repeating FRBs. 
Precise, sub-arcsecond localization of FRBs allows for unambiguous association with galaxies and the environs in which they reside. These associations inform the choice of the models invoked to explain particular FRBs, as they constrain their genesis and evolution, especially through the interplay between the progenitor and the circumburst environment (e.g. Thompson 2017, 2019; Margalit \& Metzger 2018). As an example, the evolution of the rotation measure (RM) of the repeating FRB20121102A has led to some interesting constraints on the properties of the circumburst environment (Hilmarsson et al. 2021b) and comparison with predictions (Margalit \& Metzger 2018; Piro \& Gaensler 2018).

On 2021 March 31, the Canadian Hydrogen Intensity Mapping Experiment/Fast Radio Burst (CHIME/FRB) Collaboration reported that FRB20201124A was in a very high burst activity state (CHIME/FRB Collaboration 2021). Based on this report, we proposed for and were allocated Director's Discretionary Time to observe with the upgraded Giant Metrewave Radio Telescope (uGMRT; Gupta et al. 2017) on 2021 April 5. Subsequent detections by the Commensal Real-time Australian Square Kilometre Array Pathfinder (ASKAP) Fast Transients (CRAFT) survey confined the positional uncertainty of the source to $\sim 10$ arcmin (Kumar et al. 2021a,b), which meant we could cover the entire uncertainty region with one GMRT primary beam at Band 4 (550-750 MHz). Our observations with the uGMRT, along with independent campaigns with ASKAP (Day et al. 2021a,b; Fong et al. 2021) and the Very Large Array (VLA; Law et al. 2021; Ravi et al. 2021), localized the FRB to its present coordinates.

This paper is the first in a series of three, detailing our studies of FRB20201124A with the uGMRT. Here, we describe the observations and detection of 48 bursts from FRB20201124A and present the burst properties. In Main et al. (2021, hereafter P-II), we present the first ever scintillation time-scale measurement of an FRB using combined uGMRT and 100-m Effelsberg Radio Telescope observations. Finally, in Wharton et al. (in preparation, hereafter PIII), we detail the precise localization of FRB20201124A bursts and continuum imaging of the host galaxy.

This paper is organized as follows. Section 2 describes the observations and the telescope configuration, as well as the burst detection and characterization. In Section 3, we describe the results of our DM optimization, burst localization, fluence completeness, short time-scale periodicity search, and the various properties of the bursts, such as the fluence distribution, the distribution of the burst widths, the statistics of the waiting time between bursts, and the burst spectral energies.

\section{OBSERVATIONS AND DATA}

The uGMRT observations of FRB20201124A were carried out with the Band- 4 receivers on 28 of 30 antennas available at the time, tuned to 550-750 MHz, on 2021 April 05 from 12:30 UTC to 16:30 UTC. The primary beam full width at half-maximum (FWHM) in this band is $\sim 52-38$ arcmin. At the time, the best localization radius of 10 arcmin for FRB20201124A was provided by ASKAP (Kumar et al. 2021b), which is within the 88 per cent sensitivity contour of the GMRT primary beam. We therefore recorded the incoherent array (IA; Gupta et al. 2017) beam.

For the observations reported here, we use the IA beam, in which the voltages from each antenna (and each polarization) are first detected and then added. The root-mean-square (rms) noise in the beam is hence $\sqrt{N_{\mathrm{A}}}$ times better than a single antenna. The IA beam has a field of view (FoV) as large as that of a single GMRT dish. In contrast, the phased array (PA; Gupta et al. 2017) beam is the sum of the voltages from each antenna (for the two polarizations separately) added in phase before detection. As a result, the rms noise of the PA beam is $N_{\mathrm{A}}$ times better than that of a single GMRT dish. The size of the beam is hence the same as the synthesized beam of the interferometer. The PA beam is hence $\sqrt{N_{\mathrm{A}}}$ times more sensitive than the IA beam.

The GMRT Wideband Backend FX correlator was deployed in the 200-MHz Stokes I interferometer and 8-bit beamformer mode. A Fast Fourier Transform (FFT) of every 4096 samples of the real-valued voltage time series consumes $10.24 \mu$ s of data. Sixty-four contiguous FFTs are integrated for the two polarizations individually from each antenna to obtain a 2048-channel spectrum every $655.36 \mu \mathrm{s}$, which was adequate for temporally resolving the bursts. The polarizations are finally added in quadrature to obtain Stokes I beam.

Interferometric visibility spectra were concurrently recorded with the fastest possible integration time of $671 \mathrm{~ms}$ to ensure adequate temporal sampling of the dispersed burst pulses, to aid in their imaging. Ideally, the shorter the visibility integration time, the better, as it is crucial for a clear isolation of the bursts and subsequent high-fidelity imaging. The radio source 3C 138 was used as a flux and phase calibrator due to its relative proximity to the region of pointing, resulting in relatively small slewing overheads. The array phases were tied together on 3C 138 before every 40-min scan on the target. The phase corrections for the antennas, referred to as a reference antenna, were estimated from the visibilities. The rephasing scans were also used for flux calibration. A 2-min test scan on the Crab pulsar served as a control beam for dedispersion. In all, we have $\approx 180$ min of on-target exposure.

\subsection{Detecting the bursts}

We performed a standard PRESTO ${ }^{1}$ (Ransom, Eikenberry \& Middleditch 2002) search on the IA. The data were visually inspected for radio frequency interference (RFI) and bad channels were manually flagged. The first round of dedispersion was performed with the DM of $414 \mathrm{pc} \mathrm{cm}^{-3}$ for detecting bright bursts. The data were then dedispersed with a DM of $411 \mathrm{pc} \mathrm{cm}^{-3}$, based on visual inspection of the brightest burst. The 16-bit unsigned integer (uint16) filterbanks as provided by uGMRT were converted to unsigned 8-bit (uint8) filterbanks, which were searched using standard PRESTO-based pipeline. A large amount of RFI caused the down-conversion to be sub-optimal. Therefore, in addition to above, the uint 16 filterbanks were dedispersed and searched using single_pulse_search.py. Candidates with a signal-to-noise ratio $(\mathrm{S} / \mathrm{N}) \geq 8$ (around 6000 in number) were plotted and manually vetted. A large $\mathrm{S} / \mathrm{N}$ cut-off was used owing to an excess of residual RFI in the data. In all, we detected 48 bursts over the full exposure time of $180 \mathrm{~min}$.

\subsection{Flux density calibration}

The detected bursts were isolated for calibration and fluence estimation using $1 \mathrm{~s}$ of time-frequency data centred on the dedispersed burst peak. First, the mean 'off-burst' emission was estimated in every spectral channel, which was then subtracted and divided by, to establish bandpass correction. Similarly, the corresponding phasing scan on 3C 138 and an off-calibrator scan were used to measure the deflection on the calibrator. This deflection was scaled appropriately for the spectral shape of the flux density model (Perley \& Butler

\footnotetext{
${ }^{1}$ https://github.com/scottransom/presto
} 


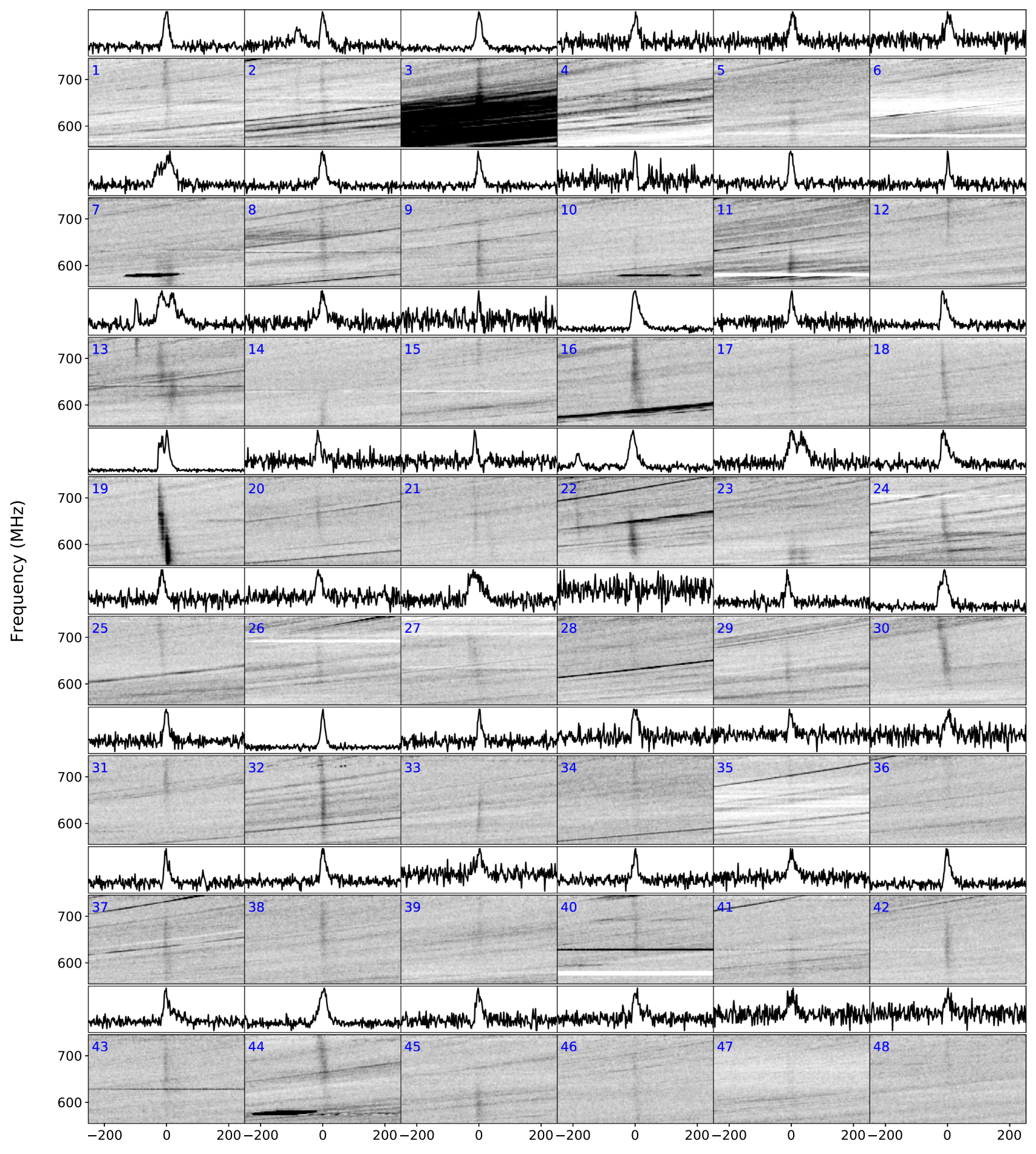

Time (ms)

Figure 1. Burst profiles and dynamic spectra in chronological order. Each panel shows the dynamic spectrum of a burst from Table 1 dedispersed to 411 pc $\mathrm{cm}^{-3}$, with the upper sub-panel showing the frequency-averaged burst profile. The dynamic spectrum has been binned $5 \times$ in frequency and $2 \times$ in time. The profile, given at the top sub-panel for each burst, is binned $3 \times$ in time. We count bursts 2,13 , and 22 as single bursts, owing to difficulty in determining if they are truly different bursts. While it is not apparent in this figure, there is a faint bridge of emission between the components in burst 22, resulting in an end-to-end width of $\sim 315 \mathrm{~ms}$. A similar argument is applied to the components in burst 2, which also is counted as single. The profiles have been cleaned for RFI by subtracting a smooth off-burst baseline through the burst. 
Table 1. Time-ordered burst measurements: The ToA is referenced to $550 \mathrm{MHz}$, the bottom of the band.

\begin{tabular}{|c|c|c|c|c|c|c|}
\hline $\begin{array}{l}\text { Burst } \\
\text { no. }\end{array}$ & $\begin{array}{c}\text { Barycentric } \\
\text { ToA } \\
\text { MJD59309 + }\end{array}$ & $\begin{array}{c}\text { Burst width } \\
\text { (zero-crossing) } \\
\text { (ms) }\end{array}$ & $\begin{array}{c}\text { Burst width } \\
\text { (boxcar equivalent) } \\
\text { (ms) }\end{array}$ & $\begin{array}{l}\text { Peak } \\
\text { flux } \\
(\mathrm{Jy})\end{array}$ & $\begin{array}{l}\text { Fluence } \\
\text { (Jy ms) }\end{array}$ & $\begin{array}{l}\text { Error } \\
\text { ( Jy ms) }\end{array}$ \\
\hline 1 & 0.54335787 & 45.6 & 17.0 & 1.1 & 18.1 & 1.7 \\
\hline 2 & 0.54591884 & 163.8 & 34.3 & 0.8 & 30.1 & 2.4 \\
\hline 3 & 0.54659070 & 91.3 & 23.0 & 1.8 & 41.3 & 1.9 \\
\hline 4 & 0.54761242 & 48.6 & 13.6 & 0.7 & 9.3 & 1.7 \\
\hline 5 & 0.55935229 & 58.5 & 17.8 & 0.7 & 12.8 & 1.7 \\
\hline 6 & 0.57848970 & 65.5 & 14.3 & 0.7 & 9.7 & 1.6 \\
\hline 7 & 0.58136744 & 126.0 & 38.4 & 0.8 & 31.9 & 2.2 \\
\hline 8 & 0.58187302 & 94.3 & 21.4 & 1.2 & 25.8 & 2.5 \\
\hline 9 & 0.58418835 & 57.5 & 16.0 & 1.3 & 20.2 & 1.8 \\
\hline 10 & 0.58529781 & 33.7 & 7.5 & 0.6 & 4.8 & 0.8 \\
\hline 11 & 0.58921682 & 36.7 & 9.5 & 1.1 & 10.5 & 1.7 \\
\hline 12 & 0.59043137 & 33.7 & 10.1 & 0.9 & 9.3 & 1.6 \\
\hline 13 & 0.59576209 & 212.3 & 59.3 & 1.0 & 58.4 & 2.9 \\
\hline 14 & 0.59626756 & 95.3 & 22.3 & 0.7 & 16.4 & 2.4 \\
\hline 15 & 0.59639863 & 19.8 & 6.1 & 0.4 & 2.6 & 0.8 \\
\hline 16 & 0.59783325 & 103.2 & 25.2 & 2.1 & 52.9 & 1.8 \\
\hline 17 & 0.59870353 & 40.7 & 11.4 & 0.7 & 7.9 & 1.6 \\
\hline 18 & 0.61101660 & 69.5 & 19.1 & 1.0 & 19.0 & 1.7 \\
\hline 19 & 0.61108629 & 75.4 & 27.9 & 3.9 & 108.0 & 1.6 \\
\hline 20 & 0.61256996 & 69.5 & 14.1 & 0.7 & 9.3 & 1.9 \\
\hline 21 & 0.61412899 & 33.7 & 7.5 & 0.7 & 5.4 & 1.4 \\
\hline 22 & 0.61761505 & 315.5 & 42.1 & 1.6 & 66.5 & 3.8 \\
\hline 23 & 0.61763151 & 112.1 & 38.3 & 0.8 & 29.0 & 2.2 \\
\hline 24 & 0.62024508 & 79.4 & 23.7 & 0.9 & 21.7 & 2.5 \\
\hline 25 & 0.62099280 & 48.6 & 11.9 & 0.8 & 9.0 & 1.5 \\
\hline 26 & 0.62315829 & 41.7 & 16.5 & 0.6 & 9.3 & 1.6 \\
\hline 27 & 0.62398148 & 86.3 & 25.4 & 0.8 & 20.4 & 2.1 \\
\hline 28 & 0.62409520 & 18.9 & 0.7 & 0.0 & 0.0 & 0.1 \\
\hline 29 & 0.62932227 & 52.6 & 13.7 & 0.7 & 9.6 & 1.8 \\
\hline 30 & 0.63120928 & 84.3 & 22.9 & 1.3 & 29.7 & 1.8 \\
\hline 31 & 0.64163927 & 59.5 & 17.2 & 0.7 & 11.9 & 1.6 \\
\hline 32 & 0.64281245 & 61.5 & 15.4 & 2.0 & 31.3 & 1.6 \\
\hline 33 & 0.64447160 & 51.6 & 13.3 & 0.8 & 10.0 & 1.5 \\
\hline 34 & 0.64848111 & 36.7 & 15.3 & 0.6 & 8.9 & 1.6 \\
\hline 35 & 0.65177353 & 43.7 & 10.7 & 0.7 & 7.4 & 1.7 \\
\hline 36 & 0.65382734 & 44.6 & 15.7 & 0.5 & 7.2 & 1.6 \\
\hline 37 & 0.65603984 & 51.6 & 13.1 & 0.9 & 12.1 & 1.7 \\
\hline 38 & 0.65638858 & 82.4 & 18.6 & 1.0 & 18.9 & 1.9 \\
\hline 39 & 0.65702755 & 68.5 & 15.6 & 0.5 & 8.5 & 1.8 \\
\hline 40 & 0.65930498 & 62.5 & 14.8 & 0.8 & 11.2 & 1.6 \\
\hline 41 & 0.66312276 & 54.6 & 18.7 & 0.7 & 12.4 & 1.9 \\
\hline 42 & 0.66338438 & 63.5 & 16.8 & 1.1 & 17.8 & 1.9 \\
\hline 43 & 0.66415983 & 93.3 & 25.1 & 0.9 & 22.2 & 1.8 \\
\hline 44 & 0.66577462 & 92.3 & 29.5 & 1.1 & 32.5 & 2.4 \\
\hline 45 & 0.67941141 & 45.6 & 15.7 & 0.7 & 11.4 & 1.9 \\
\hline 46 & 0.67978340 & 63.5 & 20.9 & 0.7 & 14.7 & 1.9 \\
\hline 47 & 0.68965288 & 42.7 & 12.1 & 0.6 & 7.0 & 1.7 \\
\hline 48 & 0.68973336 & 53.6 & 10.3 & 0.5 & 5.4 & 1.6 \\
\hline
\end{tabular}

While burst 28 was detected after RFI cleaning, its fluence could not be estimated reliably.

2017). The frequency-averaged time series of the burst and the calibrator scan were then compared to set the flux scale for the bursts, from which we read off the peak flux and estimate the fluence. The time of arrival (ToA) for each burst is measured at the peak, referred to as the lowest frequency of the band, $550 \mathrm{MHz}$. Since the localized position of the FRB is less than 2.5 arcmin away from the antenna boresight (see Section 3.2), no primary beam correction has been applied for our fluence estimates, given that the GMRT FoV at Band 4 is $\sim 40$ arcmin. The gallery of the detected bursts is shown in Fig. 1. The ToA, burst width, peak flux, fluence, and error bar on the fluence are all listed in Table 1.

\section{RESULTS AND PROPERTIES}

\subsection{DM optimization with the power spectrum}

In detecting the bursts, as well as estimating their fluences, widths, and ToAs, we use have used DM $=411 \mathrm{pc} \mathrm{cm}^{-3}$. However, as noted in Marthi et al. (2020), the DM that maximizes the peak S/N gives a profile that is degenerate with intrinsic temporal sub-structure. For bursts that consist of multiple components, a reasonable assumption to consider is that the individual components have no intrinsic drift, while there is only a bulk drift between the components (e.g. in 

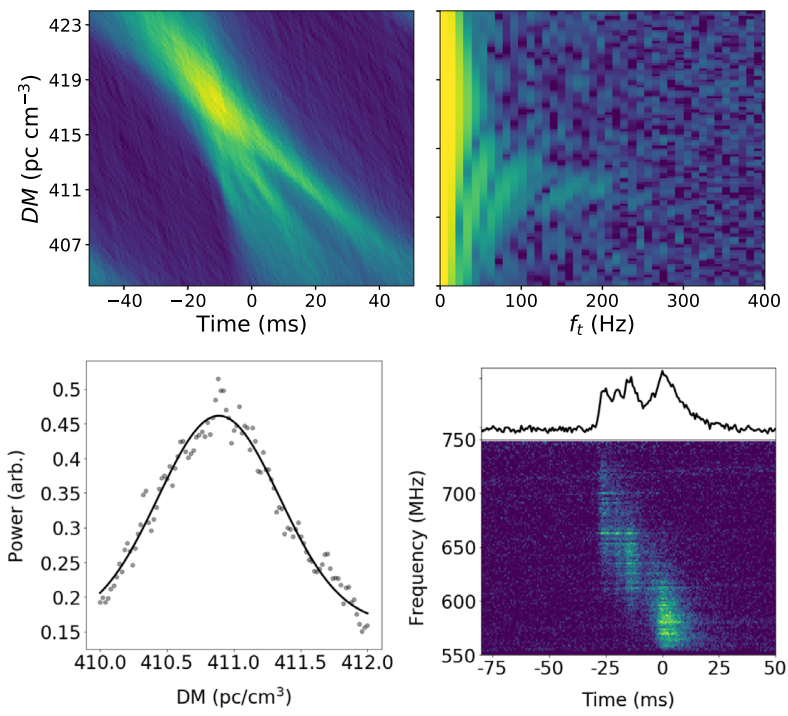

Figure 2. DM estimation from burst 19 using the SVD method. Top left-hand panel: the frequency-averaged burst profile as a function of DM. Note that the $\mathrm{S} / \mathrm{N}$ peaks at $\sim 418 \mathrm{pc} \mathrm{cm}^{-3}$, but that it compromises the sub-structure. Top right-hand panel: the power spectrum as a function of the DM. Bottom lefthand panel: $\mathrm{S} / \mathrm{N}$ of the burst power at $180 \mathrm{~Hz}$, following the steps outlined in Section 3.1. Bottom right-hand panel: the waterfall and the profile for $\mathrm{DM}=410.78 \mathrm{pc} \mathrm{cm}^{-3}$.

Hessels et al. 2019, they make a similar argument). One would expect that for the DM that aligns each component perfectly across frequency, it maximizes the total energy in the sub-structure of a frequency-averaged burst profile. We employ the same method outlined in Marthi et al. (2020) to determine the DM that maximizes the energy in the sub-structure, but we expand on it here for completeness.

We incoherently dedisperse the brightest burst for DMs between 410.00 and $412.00 \mathrm{pc} \mathrm{cm}^{-3}$ in steps of $0.01 \mathrm{pc} \mathrm{cm}^{-3}$. The steps undertaken to determine the DM that maximizes sub-structure energy are as follows: (i) A singular value decomposition (SVD) of the burst dynamic spectrum is taken. The leading mode eigenfunction of frequency for both of the on- and off-pulse, as a weighting function across the frequency, is applied to the dynamic spectrum, and the time-series data determined. (ii) An FFT of the time-series data produces the power spectrum across Doppler frequencies for both of the on- and off-pulse. The power spectrum of the off-pulse is subtracted from that of the on-pulse, giving us the noise-subtracted power spectrum. (iii) The noise-subtracted power spectrum, which we rebinned from 200 Doppler frequencies into 16 in log-scale, is examined from low to high log-Doppler frequencies to determine the cut-off Doppler frequency when the power across DM shows a Gaussian maximum. For the brightest burst, the power versus $\mathrm{DM}$ at the cut-off log-Doppler frequency $0.18 \mathrm{kHz}$ is shown in Fig. 2. (iv) A Guassian profile is fitted to the power versus DM to determine the optimal DM and the error, at the peak and the FWHM, respectively. The DM thus identified is $410.78 \pm 0.54(1 \sigma)$ pc $\mathrm{cm}^{-3}$.

We ran DM_PHASE ${ }^{2}$ (Seymour, Michilli \& Pleunis 2019), the DM optimization routine that maximizes the coherent power across the bandwidth, for comparison with our SVD-based method on the

\footnotetext{
${ }^{2}$ https://github.com/danielemichilli/DM_phase
}

brightest burst. It returned a DM of $410.33 \pm 1.15(1 \sigma) \mathrm{pccm}^{-3}$, which is in agreement within the error bars with the DM optimized by our method.

\subsection{Burst localization and persistent radio emission}

At the time of observation, the best localization (Kumar et al. 2021b) for FRB20201124A had an uncertainty of 15 arcmin in right ascension (RA) and 5 arcmin in declination (Dec.). This is well within the primary beam of the UGMRT, with excellent prospects for simultaneous localization with the visibilities. Our proposal to observe with the uGMRT focused on the localization of the bursts by using its simultaneous interferometric and beamformer capabilities. We observed with the boresight pointing at (J2000) RA: $05^{\mathrm{h}} 07^{\mathrm{m}} 55^{\mathrm{s}}$, Dec.: $+26^{\circ} 02^{\prime} 00^{\prime \prime}$.

After identifying the ToA of the bursts in the beam, the visibilities of the brightest burst (burst 19) were imaged and the burst was localized to sub-arcsecond precision (Wharton et al. 2021b). The position was consistent with the earlier VLA localization (Law et al. 2021) and subsequent localizations with the ASKAP low-band (Day et al. 2021b) and the EVN (Marcote et al. 2021). In addition, we detect persistent radio emission of $0.7 \pm 0.1 \mathrm{mJy}$ at $650 \mathrm{MHz}$ (Wharton et al. 2021a), coincident with both the burst position and the galaxy SDSS J050803.48 + 260338.0. The persistent radio emission was later found to be resolved on milliarcsecond scales (Marcote et al. 2021) and likely associated with star formation in the host galaxy (Fong et al. 2021; Ravi et al. 2021). The complete analysis and findings of the uGRMT burst localization are described in PIII. These observations provide a proof of concept for future burst localizations of active repeating FRBs with the uGMRT.

\subsection{Spectral energy}

The most energetic burst in our observations has the largest peak flux. At $108 \mathrm{Jy} \mathrm{ms}$, this bright burst is detected with an $\mathrm{S} / \mathrm{N}$ of $\sim 40$, $\sim 4 \times$ to $5 \times$ lower than ideal due to RFI. This burst would have been detected with an $\mathrm{S} / \mathrm{N}$ of $\gtrsim 200$ with the PA beam under the same RFI conditions, considering the $\sqrt{N_{\mathrm{A}}}$ boost in sensitivity accrued from co-adding the voltages in phase. At a luminosity distance of $451 \mathrm{Mpc}$ (with $h=0.7, \Omega_{\Lambda}=0.7, \Omega_{\mathrm{m}}=0.3, z=0.098 \pm 0.02$; Fong et al. 2021; Kilpatrick et al. 2021; Ravi et al. 2021), the isotropic equivalent spectral energy of burst 19 is $2.63 \times 10^{31} \mathrm{erg} \mathrm{Hz}^{-1}$. The faintest burst is $2.6 \mathrm{Jy} \mathrm{ms}$, or $6.33 \times 10^{29} \mathrm{erg} \mathrm{Hz}^{-1}$, and is still more than three orders of magnitude more energetic than the Galactic FRB SGR 1935 + 2154 (Bochenek et al. 2020; CHIME/FRB Collaboration et al. 2020b). This is roughly two orders of magnitude more than the $10 \times$ to $25 \times$ energy gap for the faintest $0.1 \mathrm{Jy} \mathrm{ms}$ burst from FRB20180916B (Marthi et al. 2020), but it is not surprising: Even for a faint $0.1 \mathrm{Jy}$ ms burst that might have been detected with the PA beam, the larger distance of FRB20201124A means that only those bursts above a threshold spectral energy of $2.43 \times 10^{28} \mathrm{erg} \mathrm{Hz}^{-1}$ are automatically selected. We note that the computed spectral energies are lower limits for two reasons: (1) Often the bursts do not occupy the full band, and (2) there is an unknown beaming factor. The key to bridging the energy gap with the Galactic FRB lies in detecting extremely close FRBs, such as FRB20200120E (Bhardwaj et al. 2021; Kirsten et al. 2021).

\subsection{Burst widths}

We define the boxcar equivalent width of a burst as the ratio of the fluence to the peak flux, measured in ms: This number therefore 


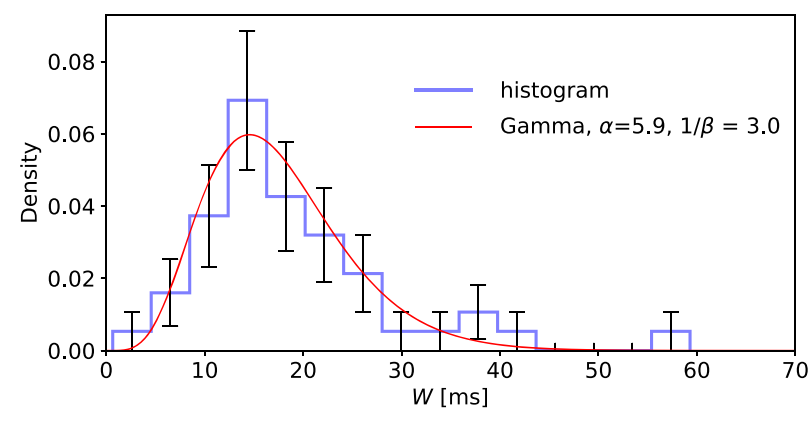

Figure 3. The histogram of the boxcar equivalent burst widths and the PDF of the best-fitting Gamma distribution $f_{\mathbf{W}}(w ; \alpha, \beta)$. The $W>35 \mathrm{~ms}$ bursts are excluded from the fit as they arise from multiple distinct components counted as a single burst.

represents the width of a boxcar window whose height is equal to the peak flux to give the measured fluence. The burst widths measured between the zero crossings of a running mean profile, obtained with a 16-bin boxcar kernel, along with the boxcar equivalent width, are given in Table 1. However, we use the equivalent width as the basis for simulating events in our completeness analysis in Section 3.6.

We do not attempt to determine the underlying probability distribution function (PDF) of the burst widths, as there are no physical models that can inform our choice. Instead, we merely attempt to derive an empirical fit to the data, to aid in the completeness analysis.

Fig. 3 shows the histogram of the burst width $W$. The errors on the histogram are binwise Poissonian. We find the best-fitting Gamma distribution for the burst width histogram, which is continuous in the random variable $W$. The Gamma distribution is a general two-parameter family of continuous distributions, of which the exponential, Chi-squared, and Erlang distributions are special cases. The PDF of the Gamma distribution is

$f_{W}(w ; \alpha, \beta)=\frac{\beta^{\alpha} w^{\alpha-1} \mathrm{e}^{-\beta w}}{\Gamma(\alpha)}$,

where $\alpha$ is the shape parameter and $\beta$ is the rate parameter. The best-fitting values are $(\alpha, 1 / \beta)=(5.9,3.0)$, obtained after excluding bursts with $W>35 \mathrm{~ms}$. The mean of the PDF is given by $\mu=$ $\alpha / \beta=17.5 \mathrm{~ms}$, the standard deviation is $7.2 \mathrm{~ms}$, and the empirically determined mean width is $\sim 17.2 \mathrm{~ms}$. The larger widths correspond to instances where multiple bursts are counted as a single event (see e.g. Fig. 1 and Table 1, bursts 2, 13, and 22).

The burst widths of FRB20201124A are, on average, larger than those from other known repeaters. The majority of bursts from repeaters detected by CHIME/FRB have durations $<25 \mathrm{~ms}$ (Pleunis et al. 2021a). We additionally note that the widths of detections of FRB20201124A at $1.4 \mathrm{GHz}$ are also unusually large (Hilmarsson et al. 2021a). Also, note that since our data are incoherently dedispersed, there is an intra-channel smearing of $\sim 2 \mathrm{~ms}$ at the lowest frequency.

\subsection{Fluence distribution}

Fig. 4 shows the cumulative burst rate function of the burst fluence, $R(>F) \propto F^{\gamma}$, which gives the rate of bursts above a fluence $F$. The power-law index, $\gamma$, was determined using a maximum-likelihood estimator (James et al. 2019, and references therein) and excluding bursts below the completeness limit. Our fit yielded the power-law index of $\gamma=-1.2 \pm 0.2$, by setting a visually identified completeness limit of $10 \mathrm{Jy} \mathrm{ms}$. We performed a more rigorous analysis with this $\gamma$ that gives a completeness limit of $7 \mathrm{Jy}$ ms (described in Section 3.6).

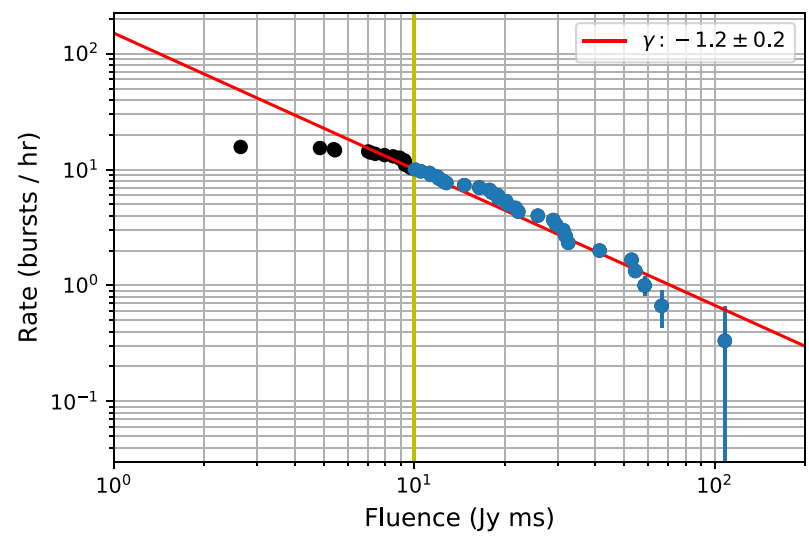

Figure 4. Cumulative burst rate function of the detected bursts. The bursts are complete up to a fluence limit of $10 \mathrm{Jy}$ ms (vertical yellow line). The red line shows the best-fitting power law excluding bursts below the completeness level. The rate function is $R(>F)=10 \mathrm{~h}^{-1}(F / 10 \mathrm{Jy} \mathrm{ms})^{-1.2}$. Only the blue data points $(F>10 \mathrm{Jy} \mathrm{ms})$ are included in the fit, while the black data points are fainter than the completeness limit.

The cumulative burst rate function inferred from our observations is

$R(>F)=10 \mathrm{~h}^{-1}(F / 10 \mathrm{Jy} \mathrm{ms})^{-1.2}$.

The power-law index determined here is shallower than that of the well-studied FRB20121102A at $1.4 \mathrm{GHz}$, where it is a steep $\gamma=-1.8 \pm 0.3$ (Gourdji et al. 2019), although there is evidence for bi-modality in this source ( $\mathrm{Li}$ et al. 2021) that cannot be well described by a single power law. CHIME/FRB determined $\gamma=$ $-1.3 \pm 0.3 \pm 0.1$ for FRB20180916B at observing frequencies comparable to ours (CHIME/FRB Collaboration et al. 2020a), which is consistent with our measurement of FRB20201124A. For comparison, Crab supergiant pulses show steeper distributions: $\gamma=$ -1.8 to -2 (e.g. Karuppusamy, Stappers \& van Straten 2010; Bera \& Chengalur 2019).

Repeating FRBs are subject to strong selection effects on $\gamma$. Since there are more faint bursts than bright ones, nearby FRBs are more likely to be detected than far away ones. For $\gamma<-1.5$, the expected distance diverges nearby, meaning we expect to be dominated by nearby FRBs, while for $\gamma>-1.5$, the event rate is dominated by far away ones. For the observed event rates to converge, we conclude that the intrinsic population has $\gamma>-1.5$ above some luminosity $L_{0}$, and $\gamma<-1.5$ below. In a flux-limited survey, most FRBs will be detected near $L_{0}$, and have an apparent $\gamma \sim-1.5$, which is indeed the case for FRB20201124A.

\subsection{Fluence completeness}

For the GMRT IA beam, the rms noise in $10 \mathrm{~ms}$ is $\sigma_{\mathrm{IA}} \approx 30 \mathrm{mJy}$. For a burst with a peak flux of $S_{\mathrm{p}}=300 \mathrm{mJy}$ and pulse width of $W=$ $10 \mathrm{~ms}$, we get a $10 \sigma$ detection:

$\mathrm{S} / \mathrm{N}_{\mathrm{IA}}=10\left(\frac{S_{\mathrm{p}}}{300 \mathrm{mJy}}\right)\left(\frac{W}{10 \mathrm{~ms}}\right)^{0.5}$.

For a fluence $F_{\mathrm{p}}=S_{\mathrm{p}} W=3 \mathrm{Jy} \mathrm{ms}$,

$\mathrm{S} / \mathrm{N}_{\mathrm{IA}}=10\left(\frac{F_{\mathrm{p}}}{3 \mathrm{Jy} \mathrm{ms}}\right)\left(\frac{W}{10 \mathrm{~ms}}\right)^{-0.5}$

and we should be able to detect 10 -ms bursts at $10 \sigma$, with brighter, narrower bursts being more likely detected. 


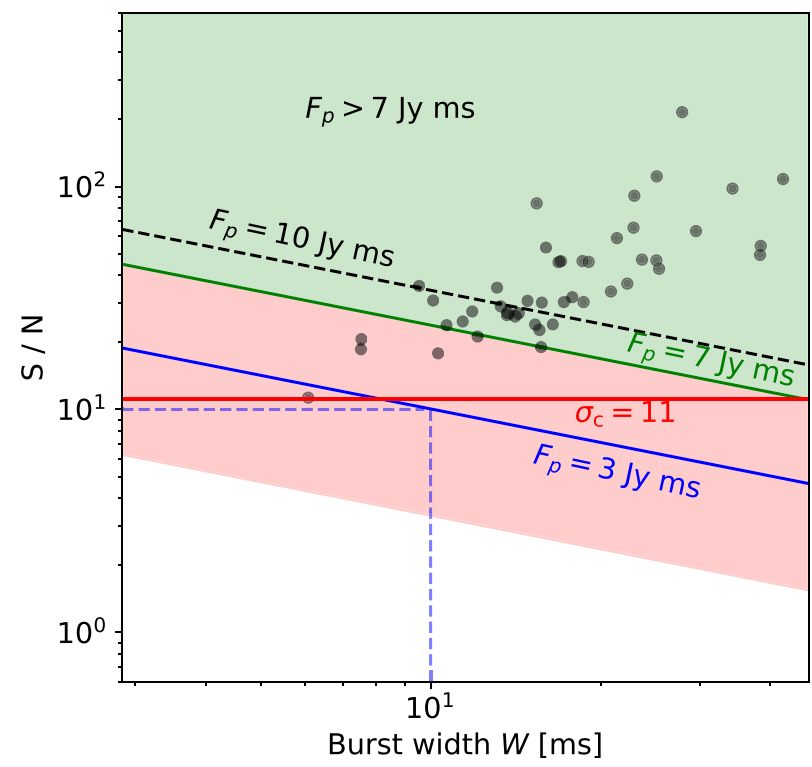

Figure 5. The completeness fluence limit is $7 \mathrm{Jy} \mathrm{ms}$. All bursts that fall in the shaded green region are detected, while only a fraction of those below the limit, in the shaded red region, are detected. The missed fraction is $\sim 80$ percent in the 1-7 Jy ms range denoted by the shaded red region. The $F_{\mathrm{p}}=3 \mathrm{Jyms}$ line is the reference fluence for a $10 \sigma$ detection of a 10-ms-wide burst, according to equation (4). The scatter points represent the detected bursts, for which the $\mathrm{S} / \mathrm{N}$ are computed according to equation (4).

The faintest burst we detect in our observations has a fluence of $2.6 \mathrm{Jy} \mathrm{ms}$, an $\sim 11 \sigma$ detection as defined by equation (4). However, the completeness fluence limit is likely much higher, which we determine as follows.

We draw a random sample of $10^{3}$ bursts from the cumulative fluence distribution of Fig. 4, using the empirically determined equation (2). The lower limit to the fluence is set to $1 \mathrm{Jyms}$. Next, we draw a random sample of $10^{3}$ burst widths given by the Gamma distribution with the best-fitting $(\alpha, \beta)$. Assuming $W$ and $F_{\mathrm{p}}$ are independent, we compute the $\mathrm{S} / \mathrm{N}$ for every pair (obtained as an outer product) using equation (4). For a cut-off $\mathrm{S} / \mathrm{N}$, defined as $\sigma_{\mathrm{c}}=$ $(\mathrm{S} / \mathrm{N}) / \sigma=11$ (see Fig. 5), we find that the lowest fluence for which bursts of all widths are detected is $\sim 7 \mathrm{Jy} \mathrm{ms}$.

We are now specifically interested in the missed fraction of bursts below the completeness limit, up to a reasonable fluence limit. We can compute this quantity as a fraction of the $F_{\mathrm{p}}<7 \mathrm{Jy}$ ms events that satisfy the $\mathrm{S} / \mathrm{N}<\sigma_{\mathrm{c}}$ as well as $F_{\mathrm{p}}>F_{\mathrm{p}}^{\text {th }}$ condition. For a fluence detection threshold $F_{\mathrm{p}}^{\text {th }}=1 \mathrm{Jyms}$, we find that our observations would have missed $\sim 80$ percent of all bursts $1<F_{\mathrm{p}}<7$ Jy ms. However, our observing conditions were far less than ideal due to the RFI susceptibility of the IA beam. The missed fraction we determine here is hence a lower limit, as we consider $10 \mathrm{Jy}$ ms as the more conservative completeness limit.

From the scatter points overlaid on the $W-\mathrm{S} / \mathrm{N}$ space in Fig. 5, it is obvious that bursts with lower widths tend to have lower fluence values. It raises the possibility that the large fraction of bursts being missed could have had intrinsically small burst widths. However, the burst widths follow a Gamma distribution, with the mean burst width being $17.5 \pm 7.2 \mathrm{~ms}$, suggesting an inherent deficiency of narrow bursts. On the other hand, it is likely that the burst width distribution itself is biased towards the wider burst population, influenced by the limited sensitivity to narrow bursts (lower fluences).

\subsection{Burst rate and arrival times}

The number of bursts detected in each scan is, respectively, 5, 12, 13, 14, and 4. The first four exposures are $40 \mathrm{~min}$ (see Fig. 6a), while the last one is $20 \mathrm{~min}$ long. Considering that all bursts above the $\sim 11 \sigma$ limit of $2.6 \mathrm{Jy} \mathrm{ms}$ have been detected, we get a burst rate of $\sim 16 \mathrm{~h}^{-1}$, which is likely to be a lower limit due to the incomplete fraction $<10 \mathrm{Jy} \mathrm{ms}$. The rate is $10 \mathrm{~h}^{-1}$ above the completeness limit of 10 Jy ms. Unlike FRB20180916B, which shows a highly variable burst rate (Marthi et al. 2020), FRB20201124A appears to burst at a more uniform rate at least up to the fluence limit of $2.6 \mathrm{Jy} \mathrm{ms.}$ A more sensitive observation with the fully phased beam, which is roughly $5 \times$ more sensitive, could reveal a population of bursts, which could revise both the overall burst rate and its uniformity. For the PA beam, given an $8 \sigma$ threshold of $0.5 \mathrm{Jy} \mathrm{ms}$, the empirically determined cumulative distribution function of equation (2) returns a phenomenal rate of $\sim 360 \mathrm{~h}^{-1}$, if the power law still holds good at the PA beam $8 \sigma$ fluence threshold. If confirmed observationally, FRB20201124A would qualify as the most prolific and active repeating FRB known as yet. Li et al. (2021) observe a peak rate of $122 \mathrm{~h}^{-1}$ but falling sharply subsequently, suggesting a variable burst rate in FRB20121102A. In FRB20201124A, it remains to be seen if the highly optimistic burst rate obtained from an extrapolation of the power law to $0.5 \mathrm{Jy} \mathrm{ms}$ as well as the uniformity over several epochs holds. It is also likely that our observations occurred at a time when FRB20201124A was in an extremely active state. Analysis of the cumulative rate distribution from different, widely spaced observations may shed more light on whether the burst rate is evolving.

The longest gap between the scans (see Fig. 6a) is $\approx 11.7 \mathrm{~min}$, while the typical gap is $8-9$ min long. This would skew the histogram of burst waiting times slightly, resulting in undercounted bursts for waiting times $\lesssim 10 \mathrm{~min}$, while overcounting ones $>10 \mathrm{~min}$, assuming a small number of bursts occurred in the scan gaps. In the absence of any priors on the underlying PDF, we cannot account for bursts missed in the rephasing intervals between the scans. One way to circumvent this difficulty is to exclude the pairs that straddle the scan gaps. This results in only a loss of 4 of the 47 waiting times, but results in a more truthful histogram. The mean waiting time between bursts, excluding the four inter-scan pairs, is $t_{\mathrm{w}} \sim 2.91 \mathrm{~min}$. Fig. 6(b) shows the histogram of the waiting times between successive bursts with the error bars obtained from the barycentred ToAs, excluding the four pairs as described above.

We ran a simulation by drawing waiting times from an exponential mass function with the empirically determined mean waiting time $t_{\mathrm{W}}$,

$f\left(t ; t_{\mathrm{w}}\right)=N_{\mathrm{b}} \frac{1}{t_{\mathrm{w}}} \mathrm{e}^{-\left(t / t_{\mathrm{w}}\right)}$,

generating $N_{\mathrm{b}}=50$ bursts in each run. These bursts were distributed within the observation as if they were observed with the same scan durations and intervals shown in Fig. 6(a). Those bursts that fell in the scan intervals, as well as at the book-end bursts in each scan, were excluded before obtaining the histogram. The mean and the error bar in each bin were determined from 1000 iterations: However, increasing the number of iterations beyond $\sim 50$ has very little effect on the mean and the Poissonian error bars as they tend to converge. We note that the simulated waiting times drawn from an exponential distribution agree well with the burst waiting time histogram, suggesting a good match of the data with the distribution. The outputs from the simulation are plotted as points with error bars. Curves for the exponential mass function are overplotted for $40 \leq$ $N_{\mathrm{b}} \leq 50$ (see Fig. 6b). 


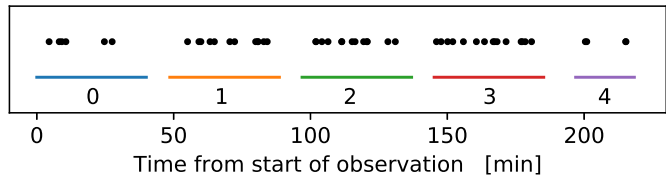

(a) Burst time positions within each scan

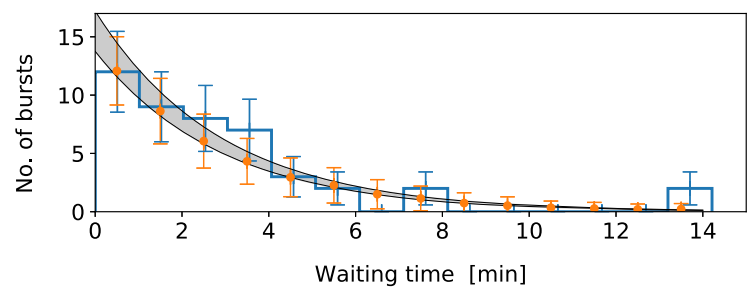

(b) Burst waiting time

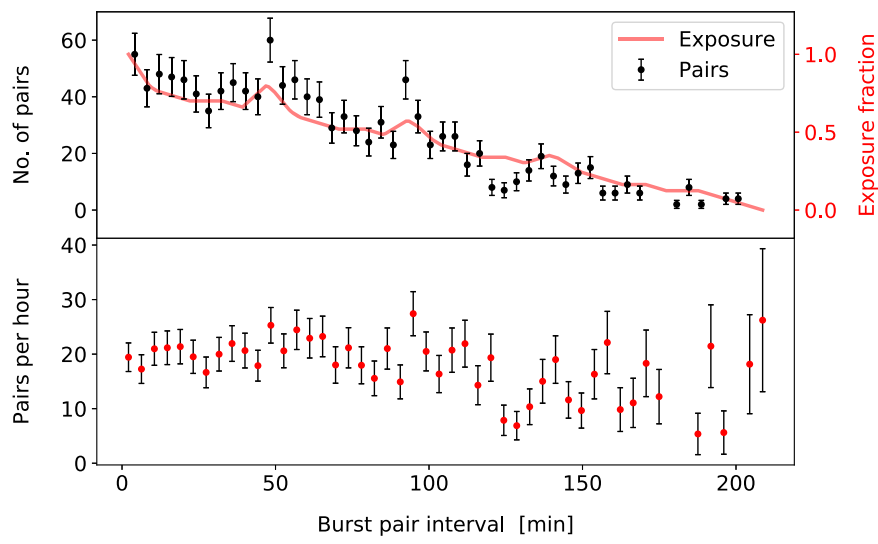

(C) Burst pair interval

Figure 6. (a) The occurrence of the bursts within each scan. The first four scans are $\approx 40$ min long each, and the last one is 20 min, with $5,12,13,14$, and 4 bursts, respectively. (b) Histogram of the burst waiting time, with the Poissonian error bars. The histogram of simulated bursts is shown as points with error bars. The shaded region represents the family of exponential mass function curves given by equation (5) for $40 \leq N_{\mathrm{b}} \leq 50$ with $\lambda=1 / t_{\mathrm{W}}$ (where $t_{\mathrm{w}}=2.91$ min), from which the simulated bursts are drawn. (c) Burst pair intervals, before (top) and after (bottom) correcting for relative exposure. The error bars are Poissonian.

An exponential distribution for the waiting time suggests an underlying Poisson point process with a rate parameter $\lambda=1 / t_{\mathrm{w}}$. This is expected for bursts detected within a single observation, as seen for FRB20121102A (Cruces et al. 2021), which is known to follow a Weibull distribution over a longer time with a clustering factor of $k=0.34_{-0.05}^{+0.06}$ (Oppermann, Yu \& Pen 2018). With only 48 bursts, of which only a fraction is complete (30 of 48 bursts, above $10 \mathrm{Jy} \mathrm{ms}$ ), there may be pitfalls to modelling the burst waiting time distribution. At this juncture, we limit our discussion to the empirical fit of the distribution. The statistics should benefit significantly from sensitive PA beam detections with a much deeper completeness limit. In addition, continued monitoring over long periods should assist in identifying temporal clustering behaviour if any, considering that it has recently entered a state of heightened activity.

Li et al. (2021) report waiting time statistics for FRB20121102A using a collection of $\sim 1600$ bursts. They find the waiting time between bursts to be well fitted by a lognormal distribution. Their waiting time histogram shows two peaks, with the largest mean waiting time being $\sim 70 \pm 12 \mathrm{~s}$, which they consider to be an upper limit to any periodicity, and a separate $\sim 220 \pm 100 \mathrm{~s}$ limit to the periodicity of the high-energy $\left(>3 \times 10^{38} \mathrm{erg}\right.$ ) bursts. They conclude that these values are consistent with the mean values for the respective samples, and therefore consistent with underlying stochasticity ( $\mathrm{Li}$ et al. 2021). While we do find a few bursts with multiple components separated by $\sim 90$ or $\sim 180 \mathrm{~s}$, we do not have sufficient sensitivity to identify them as distinct and independent bursts. Such closely separated bursts deserve keen attention to determine if they truly arise from a different distribution peaking at a shorter mean waiting time, such as seen for FRB20121102A by Li et al. (2021).

In addition, we derive a histogram of the pairwise intervals between all burst pairs, to investigate any trends for a preferred separation time, or multiples thereof, between bursts that might hint at longer periodicity time-scales or burst rate modulation. The raw histogram is shown in the top panel of Fig. 6(c). This, however, is biased by the relative exposure between pairs. The relative exposure as a function of burst pair separation is obtained as the autocorrelation function (ACF) of the scan windows (see Fig. 6a), scaled for the number of burst pairs at zero separation (which is the total number of detected bursts). Obviously, shorter pair separations have a higher relative exposure than longer ones. The peaks in the relative exposure correspond to the $\sim 45$-min interval between successive scan start times. The normalized histogram is given in the bottom panel. We find no evidence for a preferred pair separation time that might indicate longer periodicities or rate modulation of $\sim$ minutes timescales.

\subsection{Spectro-temporal drift}

The IA beam is very susceptible to RFI. Broad-band, zero-DM RFI bursts show a characteristic reverse dispersion sweep after dedispersion. An SVD of the contaminated, but dedispersed, burst dynamic spectrum breaks the reverse-swept RFI features and distributes it across a very large number of modes. While the total energy in the RFI is very high, often much more than the burst itself, it does not dominate the rank-ordered singular values. The dominant orthogonal modes of the dedispersed burst dynamic spectrum can thus purely represent the burst. This filtering method works well for bursts with reasonably high $\mathrm{S} / \mathrm{N}$, but becomes progressively less effective with deteriorating $\mathrm{S} / \mathrm{N}$. This is not surprising, as the ability to exclude the reverse-swept RFI modes depends on their rank ordering in the presence of the compact representation of the true burst modes.

As an example, in Fig. 7, we show the 2D ACF and the estimated drift rate for the brightest burst, as it has more than twice the $\mathrm{S} / \mathrm{N}$ of any other burst. For this burst (burst 19), we find that, using the SVD mode filtering described above, the first six modes adequately represent the burst, allowing us to exclude all the subsequent modes and thus nearly fully eliminate the swept broad-band RFI features. The reconstructed dynamic spectrum was used to obtain the $2 \mathrm{D} \mathrm{ACF}$, allowing us to estimate a drift rate of $\Delta f / \Delta t=-2 \mathrm{MHz} \mathrm{ms}^{-1}$. Other bursts show drift rates between $\sim-0.75$ and $\sim-20 \mathrm{MHz} \mathrm{ms}^{-1}$.

All the bursts in our sample show a downward drift or a 'sad trombone' effect, as is known for repeaters (Hessels et al. 2019; Caleb et al. 2020; Chawla et al. 2020), although some anomalous drifting behaviour has been seen in FRB20180916B (Pleunis et al. 2021b). In Marthi et al. (2020), we see two instances of potential upward (or positive) drift in FRB20180916B, but it is not clear if these are from the same or separate bursts. Platts et al. (2021) consider the possibility of such upward drift arising from lensing events in FRB20121102A. 


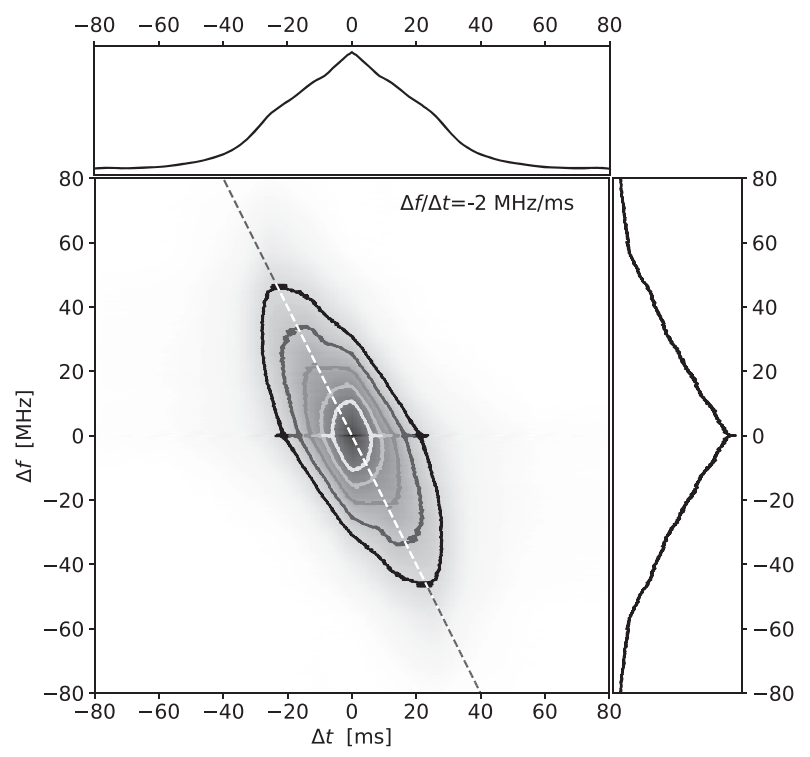

Figure 7. $2 \mathrm{D}$ ACF of burst 19, used for measuring the drift rate. The upper and the right-hand panels show, respectively, the frequency- and timeaveraged $2 \mathrm{D} \mathrm{ACF}$.

We defer an analysis of the drift rate versus burst width relation to a future paper, after including more sensitive burst detections. Chamma et al. (2021) posit the linear relation between the quantities as a possible universal relationship for repeating FRBs. Hilmarsson et al. (2021a) find the relationship between drift rates and burst widths in 1.4-GHz Effelsberg Radio Telescope observations to be consistent with the results of Chamma et al. (2021) except for an offset. The primary constraint for a similar but robust analysis of the sample presented in this paper is the unfavourable RFI contamination of the bursts, especially the weaker ones.

\subsection{Scattering and scintillation}

Fig. 8 shows the dynamic spectrum of burst 9 on the left, that of burst 16 in the middle, and that of the brightest burst (19) on the right. These bursts have a clearly identifiable decaying exponential profile: We choose bursts 9 and 16 particularly for their apparent lack of spectro-temporal drift. We consider the low-frequency portion of the bursts, where a single component of the multicomponent burst 19 is isolated by defining a cut-off frequency (here, $575 \mathrm{MHz}$ ). The other two bursts have a single component, but the cut-off frequency is defined and the analysis is done identically for consistency. The bottom panel shows the frequency-averaged (550-575 MHz) profile, which shows what appears to be an exponential tail. We fit to all the three bursts a Gaussian convolved with a decaying exponential, but constraining the decay time constant to be identical. If we interpret this as a scattering tail, we can set an upper limit of $\tau_{\mathrm{sc}} \leq 11.1 \mathrm{~ms}$ to the scattering time. The scattering time $\tau_{\mathrm{s}}$ is measured as the time constant of the decaying exponential $\mathrm{e}^{-t / \tau_{\mathrm{s}}}$, which includes the uncorrected 2-ms dispersion delay in a single $96-\mathrm{kHz}$ channel at the lowest frequency. The error bar in each burst shown in Fig. 8 is taken as the half-width at half-maximum of a Gaussian of appropriate amplitude.

In addition, the bandpass-normalized dynamic spectrum shows scintillation, evident from the peaks in the $t<0$ and $t>0$ spectra of burst 19: We measure a scintillation bandwidth of $0.1-0.2 \mathrm{MHz}$ at $550-750 \mathrm{MHz}$. Some of these features appear to be blended at the lower part of the band, whereas they appear to be adequately resolved at $\gtrsim 620 \mathrm{MHz}$. At the frequencies at which we observe, the scattering tail appears to be scintillating with a characteristic bandwidth of $\sim 0.1 \mathrm{MHz}$ but barely resolved: This might be an overestimate as the features appear to be blended. We hence have a case analogous to that of Masui et al. (2015), where the scattering is thought to occur in the host galaxy, while the scatter-broadened burst still appears 'unresolved' at a screen in the Milky way, as the scintillation appears constant throughout the scattering tail. We present a more detailed analysis of the scattering and scintillation in P-II, combining the measurements obtained from these Band-4 uGMRT and 100-m Effelsberg Radio Telescope $L$-band observations.

It is necessary to distinguish the scattering time constant from the scintillation bandwidth. In our case, the scattering time constant is not inversely related to the measured scintillation bandwidth, leading to our hypothesis that the two measurements are distinct and the two phenomena are mutually independent. In fact, the scattering time constant is roughly two orders of magnitude larger than that expected from the scintillation bandwidth. P-II develops the hypothesis that the scattering arises from a screen that is located much closer to the FRB, possibly within the host galaxy, and that the scintillation is being imparted by a scattering screen in the Milky Way.

Importantly, the fact that both scattering and scintillation are seen in this FRB allows us to place constraints on the locations of the screens and measure a velocity of the Milky Way scattering screen. The inferred scattering screen transverse velocity is $30-40 \mathrm{~km} \mathrm{~s}^{-1}$ for a screen located at $2 \mathrm{kpc}$, and much smaller for a $0.4-\mathrm{kpc}$ screen, when the velocity of the scintillation pattern has a larger contribution from the Earth's velocity. For a screen located so close to the Earth, its large contribution to the scintillation velocity is expected to manifest as a strong annual modulation of the scintillation time-scale. If the scattering arises at the host galaxy, we expect the scattering timescale to be constant throughout the annual scintillation modulation cycle.

\subsection{Periodicity search on small time-scales}

A PRESTO search for periodicities on short time-scales was done with acceleration and jerk on the full dedispersed data, as well as on multiple 10- and 20-min segments. No significant candidates were detected. Additionally, a fast folding algorithm (FFA) search was performed on the IA beam data using RIPTIDE ${ }^{3}$ (Morello et al. 2020). However, that again revealed no significant candidates. Some bursts show multiple peaks, as in bursts 2, 13, and 22. It is not clear if these are distinct components of the same burst or are different bursts, due to the poor $\mathrm{S} / \mathrm{N}$ of the IA beam. Their separations appear to be $\sim 90$ or $\sim 180 \mathrm{~ms}$. We verified through an autocorrelation analysis that the separations have no periodic relationship between the bursts.

The detection of periodicity in the range of typical pulsar periods in FRBs would be unassailable evidence for a neutron star origin for the emission and repetition. While longer periodicities of $\sim$ several days, such as known for FRB20180916B (CHIME/FRB Collaboration et al. 2020a), rely on models like orbital motion (Dai et al. 2016; Ioka \& Zhang 2020) or precession (Levin, Beloborodov \& Bransgrove 2020), smaller periodicities in the $1 \mathrm{~ms}$ to $1 \mathrm{~s}$ range are thought to arise from rotation. The CHIME/FRB Collaboration et al. (2021) have discovered multiple bursts, which appear clustered with sub-second separations, from FRB20191221A ( 217 ms), FRB20210206A ( 3 ms), and FRB20210213A ( 11 ms), consistent

\footnotetext{
${ }^{3}$ https://github.com/v-morello/riptide
} 

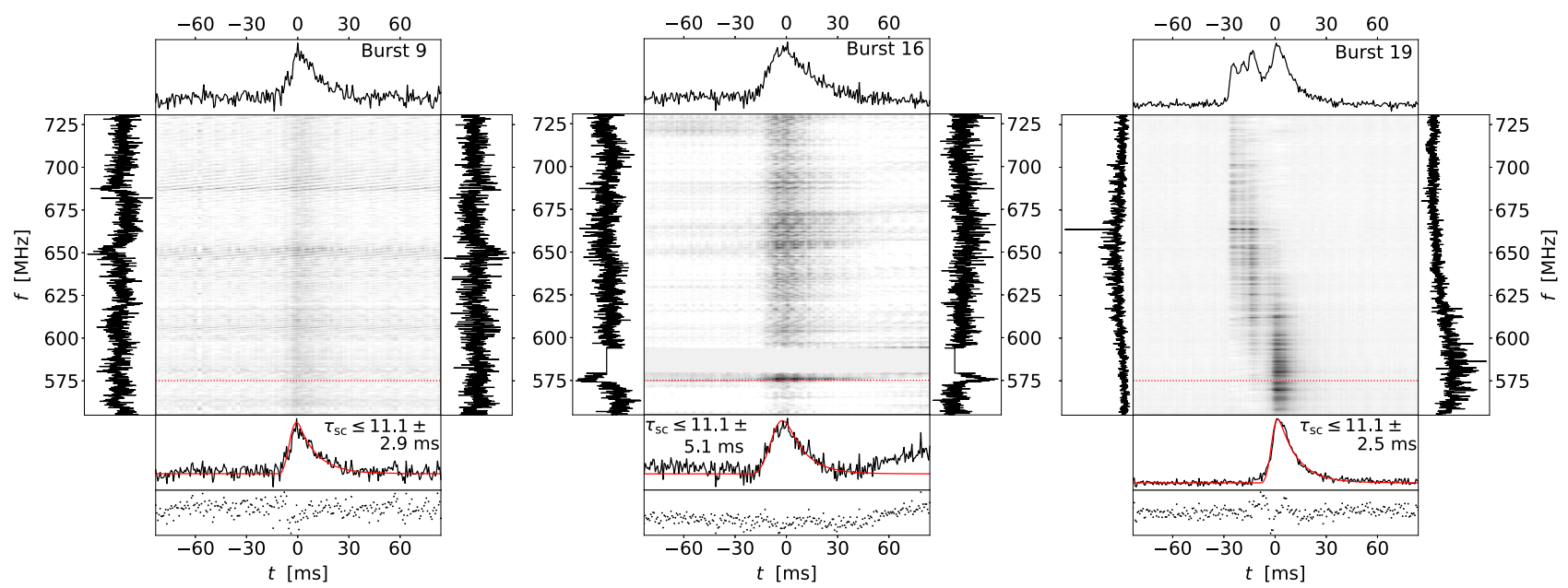

Figure 8. The SVD-filtered dynamic spectra of bursts 9, 16, and 19, dedispersed to $411 \mathrm{pc} \mathrm{cm}^{-3}$ and binned $4 \times$ in frequency for plotting. For all the three bursts, the top panel shows the mean profile integrated between 554 and $731 \mathrm{MHz}$. The bottom panel shows the low-band profile cut-off at $575 \mathrm{MHz}$. The red curve is the best fit obtained by convolving a Gaussian with a decaying exponential, giving a scattering time constant of $11.1 \mathrm{~ms}$ along with the respective $1 \sigma$ error bar, which are 2.9, 5.1, and $2.5 \mathrm{~ms}$, respectively, for bursts 9,16 , and 19. Below this panel are the residuals of the fit. The left- and right-hand sub-panels, respectively, show the burst spectrum at $t<0$ and $t>0$.

with typical periods expected for magnetospheric emission. This provides strong motivation for follow-up observations of active repeaters like FRB20201124A with longer exposures, specifically with the ability to track the source in the sky. Observations such as those described here have the potential to detect many more such clustered bursts.

In this context, it is interesting to note the possibility of ultralongperiod (ULP) magnetars as FRB candidates. Beniamini, Wadiasingh \& Metzger (2020) invoke a simple long rotation period explanation for the $\sim 16$-d periodicity of FRB20180916B. In their model, ULP magnetars arise from three distinct possibilities, such as mass-loaded charged-particle winds, loss of angular momentum due to kicks from giant flares, and long-lasting accretion discs (Beniamini et al. 2020), citing the example of the Galactic magnetar candidate with a $\sim 7$-h period, 1E 161348-5055. However, they note that such progenitors would be extremely rare, accounting for the paucity of scales of periodicity similar to FRB20180916B.

\section{SUMMARY AND CONCLUSIONS}

We report the observations at Band 4 of FRB20201124A with the upgraded GMRT and the detection of 48 bursts in $3 \mathrm{~h}$ of exposure. These observations were carried out with the dual purpose of localizing the FRB as well as detecting associated persistent emission, based on the initial, non-interferometric localization reported by ASKAP (Kumar et al. 2021b). This necessitated only a single pointing with the GMRT. We localize the FRB and detect persistent emission at $650 \mathrm{MHz}$.

We estimate the DM that maximizes the frequency-averaged sub-structure energy to be $410.78 \pm 0.54 \mathrm{pc} \mathrm{cm}^{-3}$, similar to that obtained from DM_PHASE, $410.33 \pm 1.15 \mathrm{pc} \mathrm{cm}^{-3}$. Both these DMs are consistent with the fiducial value of $411 \mathrm{pc} \mathrm{cm}^{-3}$ used throughout this paper. A larger number of high-S/N bursts from high-sensitivity observations with the PA beam are essential to constrain any burstto-burst DM variability within the observation, or for searching for systematic trends in DM variability over longer time-scales (e.g. Hilmarsson et al. 2021b, seen in FRB20121102A). We find that the mean burst width is $17.5 \pm 7.2 \mathrm{~ms}$, but find that they are larger than the widths for other known repeaters. The isotropic equivalent burst spectral energies are $\sim 10^{29}-10^{31} \mathrm{erg} \mathrm{Hz}^{-1}$.

The bursts range in fluence from 2.6 to $108 \mathrm{Jy} \mathrm{ms}$, following a power-law distribution with an index of $\gamma=-1.2 \pm 0.2$ and an overall burst rate of $16 \mathrm{~h}^{-1}$ for these observations, which is a lower limit. We estimate a completeness limit of $10 \mathrm{Jy} \mathrm{ms}$ for these observations, after empirically modelling the burst width distribution. Our observations might have missed $\gtrsim 80$ per cent of the bursts in the fluence range 1-10 Jy ms. The burst waiting time distribution agrees broadly with an exponential distribution with a mean waiting time of $t_{\mathrm{W}} \sim 2.9 \mathrm{~min}$, suggesting an underlying Poisson point process that is expected for bursts occurring within a short observation. There is no evidence for longer (minute to several minutes) periodicity or, more specifically, burst rate modulation with those characteristic timescales, inferred from the distribution of pairwise burst intervals in our observations. More sensitive observations with a tighter fluence completeness limit might revise these statistics considerably.

With a $\mathrm{DM}=411 \mathrm{pc} \mathrm{cm}^{-3}$, we measure a range of bulk spectrotemporal drift rates between -0.75 and $-20 \mathrm{MHz} \mathrm{ms}^{-1}$. We measure an upper limit to the scattering time of $\leq 11.1 \mathrm{~ms}$, as well as detect scintillation across frequency. No small time-scale, pulsarlike periodicity was detected in acceleration and jerk searches as well as with FFA.

The simultaneous localization of FRB20201124A adds strength to the proof-of-concept method adopted from our earlier work (Marthi et al. 2020). This could serve as a potential model for all our future localization and follow-up of unlocalized repeating FRBs.

More sensitive PA beam observations will likely yield an order of magnitude higher burst rate, if FRB20201124A continues in its present active state. This holds enormous promise for detailed studies of its polarization properties that would allow us to test between competing emission models, as well as study any systematic evolution of RM as reported in Hilmarsson et al. (2021b). Regular long-term monitoring would enable us to construct a putative trend for an annual scintillation time-scale modulation, and hence to locate and study the Galactic scattering screen responsible for the scintillation. Variation or modulation of burst rate or activity over longer timescales would have to be adequately sampled to search for evidence 
for clustering, as is known for FRB20121102A. Finally, although we have reported only radio observations, extremely active, localized repeating FRBs such as FRB20201124A are the best targets for extended multiwavelength campaigns that can considerably advance our current understanding of the origins of FRBs.

\section{ACKNOWLEDGEMENTS}

We thank the anonymous reviewer for comments, which have helped improve the manuscript. VRM thanks Jayaram Chengalur and Apurba Bera for insightful discussions. We thank the staff of the GMRT who have made these observations possible. GMRT is run by the National Centre for Radio Astrophysics of the Tata Institute of Fundamental Research. VRM acknowledges the support of the Department of Atomic Energy, Government of India, under project no. 12-R\&D-TFR-5.02-0700. We acknowledge the use of the CHIME/FRB Public Database, provided at https://www.chimefrb.ca/ by the CHIME/FRB Collaboration. We also acknowledge the support of the Natural Sciences and Engineering Research Council of Canada (NSERC) (funding reference number RGPIN2019-067, CRD 523638-201). We receive support from the Ontario Research Fund - Research Excellence Program (ORF-RE), Canadian Institute for Advanced Research (CIFAR), Canadian Foundation for Innovation (CFI), Simons Foundation, Thoth Technology Inc., and Alexander von Humboldt Foundation. Part of this research was carried out at the Jet Propulsion Laboratory, California Institute of Technology, under a contract with the National Aeronautics and Space Administration. LGS is a Lise Meitner Independent Max Planck Research Group Leader and acknowledges support from the Max Planck Society.

\section{DATA AVAILABILITY}

The data underlying this paper will be shared on reasonable request to the corresponding authors.

\section{REFERENCES}

Beniamini P., Wadiasingh Z., Metzger B. D., 2020, MNRAS, 496, 3390

Bera A., Chengalur J. N., 2019, MNRAS, 490, L12

Bhardwaj M. et al., 2021, ApJ, 910, L18

Bochenek C. D., Ravi V., Belov K. V., Hallinan G., Kocz J., Kulkarni S. R., McKenna D. L., 2020, Nature, 587, 59

Caleb M. et al., 2020, MNRAS, 496, 4565

Chamma M. A., Rajabi F., Wyenberg C. M., Mathews A., Houde M., 2021, MNRAS, 507, 246

Chawla P. et al., 2020, ApJ, 896, L41

CHIME/FRB Collaboration, 2021, Astron. Telegram, 14497, 1

CHIME/FRB Collaboration et al., 2020a, Nature, 582, 351

CHIME/FRB Collaboration et al., 2020b, Nature, 587, 54

Cruces M. et al., 2021, MNRAS, 500, 448

Dai Z. G., Wang J. S., Wu X. F., Huang Y. F., 2016, ApJ, 829, 27

Day C. K., Bhandari S., Deller A. T., Shannon R. M., Moss V. A., 2021a, Astron. Telegram, 14515, 1

Day C. K., Bhandari S., Deller A. T., Shannon R. M., ASKAP-CRAFT Survey Science Project, 2021b, Astron. Telegram, 14592, 1

Fong W.-f. et al., 2021, ApJ, 919, L23
Gourdji K., Michilli D., Spitler L. G., Hessels J. W. T., Seymour A., Cordes J. M., Chatterjee S., 2019, ApJ, 877, L19

Gupta Y. et al., 2017, Curr. Sci., 113, 707

Hessels J. W. T. et al., 2019, ApJ, 876, L23

Hilmarsson G. H., Spitler L. G., Main R. A., Li D. Z., 2021a, preprint (arXiv:2107.12892)

Hilmarsson G. H. et al., 2021b, ApJ, 908, L10

Ioka K., Zhang B., 2020, ApJ, 893, L26

James C. W., Ekers R. D., Macquart J. P., Bannister K. W., Shannon R. M., 2019, MNRAS, 483, 1342

Karuppusamy R., Stappers B. W., van Straten W., 2010, A\&A, 515, A36

Kilpatrick C. D., Fong W., Prochaska J. X., Tejos N., Bhandari S., Day C. K., 2021, Astron. Telegram, 14516, 1

Kirsten F. et al., 2021, preprint (arXiv:2105.11445)

Kumar P., Shannon R. M., Moss V., Qiu H., Bhandari S., 2021a, Astron. Telegram, 14502, 1

Kumar P., Shannon R. M., Keane E., Moss V. A., Askap-Craft Survey Science Project, 2021b, Astron. Telegram, 14508, 1

Law C., Tendulkar S., Clarke T., Aggarwal K., Bethapudy S., 2021, Astron. Telegram, 14526, 1

Levin Y., Beloborodov A. M., Bransgrove A., 2020, ApJ, 895, L30

Li D. et al., 2021, Nature, 598, 267

Main R. A., Hilmarsson G. H., Marthi V. R., Spitler L. G., Wharton R. S., Bethapudi S., Li D. Z., Lin H. H., 2021, preprint (arXiv:2108.00052) (P-II)

Majid W. A. et al., 2021, ApJ, 919, L6

Marcote B. et al., 2021, Astron. Telegram, 14603, 1

Margalit B., Metzger B. D., 2018, ApJ, 868, L4

Marthi V. R., Gautam T., Li D. Z., Lin H. H., Main R. A., Naidu A., Pen U. L., Wharton R. S., 2020, MNRAS, 499, L16

Masui K. et al., 2015, Nature, 528, 523

Morello V., Barr E. D., Stappers B. W., Keane E. F., Lyne A. G., 2020, MNRAS, 497, 4654

Nimmo K. et al., 2021, preprint (arXiv:2105.11446)

Oppermann N., Yu H.-R., Pen U.-L., 2018, MNRAS, 475, 5109

Perley R. A., Butler B. J., 2017, ApJS, 230, 7

Piro A. L., Gaensler B. M., 2018, ApJ, 861, 150

Platts E., Weltman A., Walters A., Tendulkar S. P., Gordin J. E. B., Kandhai S., 2019, Phys. Rep., 821, 1

Platts E. et al., 2021, MNRAS, 505, 3041

Pleunis Z. et al., 2021a, preprint (arXiv:2106.04356)

Pleunis Z. et al., 2021b, ApJ, 911, L3

Rajwade K. M. et al., 2020, MNRAS, 495, 3551

Ransom S. M., Eikenberry S. S., Middleditch J., 2002, AJ, 124, 1788

Ravi V. et al., 2021, preprint (arXiv:2106.09710)

Scholz P. et al., 2016, ApJ, 833, 177

Seymour A., Michilli D., Pleunis Z., 2019, Astrophysics Source Code Library, record ascl: 1910.004

Spitler L. G. et al., 2016, Nature, 531, 202

The CHIME/FRB Collaboration et al., 2021, preprint (arXiv:2107.08463)

Thompson C., 2017, ApJ, 844, 162

Thompson C., 2019, ApJ, 874, 48

Wharton R. et al., 2021a, Astron. Telegram, 14529, 1

Wharton R. et al., 2021b, Astron. Telegram, 14538, 1

Zhang Y. G., Gajjar V., Foster G., Siemion A., Cordes J., Law C., Wang Y., 2018, ApJ, 866, 149

This paper has been typeset from a $\mathrm{T}_{\mathrm{E}} \mathrm{X} / \mathrm{LAT} \mathrm{E}$ file prepared by the author. 